\title{
ARTICLE Herpes simplex virus-binding IgG traps HSV in human cervicovaginal mucus across the menstrual cycle and diverse vaginal microbial composition
}

Holly A. Schroeder ${ }^{1}$, Kenetta L. Nunn ${ }^{1,2}$, Alison Schaefer ${ }^{1}$, Christine E. Henry ${ }^{1}$, Felix Lam ${ }^{1}$, Michael H. Pauly ${ }^{3}$, Kevin J. Whaley ${ }^{3}$, Larry Zeitlin ${ }^{3}$, Mike S. Humphrys ${ }^{4}$, Jacques Ravel ${ }^{4,5}$ and Samuel K. Lai ${ }^{1,2}$

IgG possesses an important yet little recognized effector function in mucus. IgG bound to viral surface can immobilize otherwise readily diffusive viruses to the mucin matrix, excluding them from contacting target cells and facilitating their elimination by natural mucus clearance mechanisms. Cervicovaginal mucus (CVM) is populated by a microbial community, and its viscoelastic and barrier properties can vary substantially not only across the menstrual cycle, but also in women with distinct microbiota. How these variations impact the "muco-trapping" effector function of IgGs remains poorly understood. Here we obtained multiple fresh, undiluted CVM specimens ( $n=82$ unique specimens) from six women over time, and employed high-resolution multiple particle tracking to quantify the mobility of fluorescent Herpes Simplex Viruses (HSV-1) in CVM treated with different HSV-1-binding lgG. The IgG trapping potency was then correlated to the menstrual cycle, and the vaginal microbial composition was determined by 16 s rRNA. In the specimens studied, both polyclonal and monoclonal HSV-1-binding IgG appeared to consistently and effectively trap HSV-1 in CVM obtained at different times of the menstrual cycle and containing a diverse spectrum of commensals, including G. vaginalis-dominant microbiota. Our findings underscore the potential broad utility of this "muco-trapping" effector function of IgG to reinforce the vaginal mucosal defense, and motivates further investigation of passive immunization of the vagina as a strategy to protect against vaginally transmitted infections.

Mucosal Immunology (2018) 11:1477-1486; https://doi.org/10.1038/s41385-018-0054-z

\section{INTRODUCTION}

Mucus that coats the vaginal epithelium is primarily secreted by goblet cells in the cervix, which flows out of the cervical os by gravitational and/or intra-abdominal pressure driven flow; ${ }^{1,2}$ we call this cervicovaginal mucus (CVM) to reflect its physiological origins. CVM serves not only as a lubricant that reduces shearinduced damage to the vaginal epithelium during coitus, but also as a physical barrier that excludes pathogens in semen from immediately contacting the epithelium following ejaculation. ${ }^{3,4}$ In addition, CVM harbors a broad array of antimicrobial peptides and proteins including immunoglobulins that comprise the innate and adaptive mucosal immune defense against sexually transmitted pathogens. ${ }^{3-5}$ Thus, slowing or immobilizing viruses in CVM can not only directly reduce the pathogen load reaching target cells in the epithelium and facilitate elimination from the vagina by mucus flow to external genitalia, but also allow for more complete inactivation by innate and adaptive immune factors. Together, these functions offer the potential to block initial infections altogether.

There is a considerable interest in reinforcing the diffusional barrier properties of mucus against infections, particularly since blocking transmissions at the portal of entry minimizes the risks of systemic spread of infections. Despite the abundance of IgG in $\mathrm{CVM}^{6,7}$ the use of $\mathrm{lgG}$ to reinforce the diffusional barrier properties of CVM against pathogens was long overlooked due to the seemingly negligible affinity between individual IgG molecules and mucins. ${ }^{8-11}$ Indeed, diffusion of IgG in human mid-cycle endocervical mucus is only slowed by $\sim 10 \%$ compared to in buffer, $^{12,} 13$ implying that the affinity is so weak that most individual lgG remains unbound to mucins at any moment in time. Nevertheless, multiple IgG can accumulate on the same virion or bacterium and confer substantial binding avidity between mucins or other mucus constituents and lgG-pathogen complexes, akin to a Velcro patch with individually weak hooks. We have shown that Herpes-specific IgG can effectively trap individual Herpes Simplex Viruses (HSV-1) in CVM at sub-neutralizing concentrations via multiple transient Fc-mucin bonds. ${ }^{14}$ Importantly, trapping HSV in the mucus alone appears to offer effective protection against vaginal Herpes transmission in vivo by excluding the trapped particles from contacting the vaginal epithelium. ${ }^{11,14}$

The ability of virus-specific IgG to trap virions in the mucus is undoubtedly influenced by the rigidity and microstructure of the mucin mesh network, as well as the interactions between IgG and mucins. A large body of literature has correlated the biochemical

\footnotetext{
'Division of Pharmacoengineering and Molecular Pharmaceutics, Eshelman School of Pharmacy, University of North Carolina-Chapel Hill, Chapel Hill, NC 27519, USA; ${ }^{2}$ UNC/NCSU Joint Department of Biomedical Engineering, University of North Carolina-Chapel Hill, Chapel Hill, NC 27519, USA; ${ }^{3}$ Mapp Biopharmaceutical Inc, San Diego, CA 92121, USA;

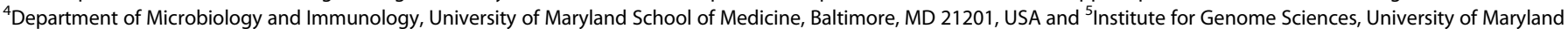
School of Medicine, Baltimore, MD 21201, USA

Correspondence: Samuel K. Lai (lai@unc.edu)
}

Received: 6 October 2017 Revised: 29 May 2018 Accepted: 31 May 2018

Published online: 9 July 2018 


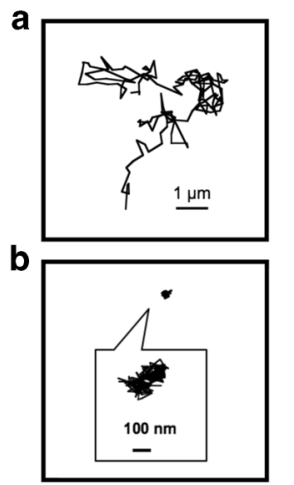

c

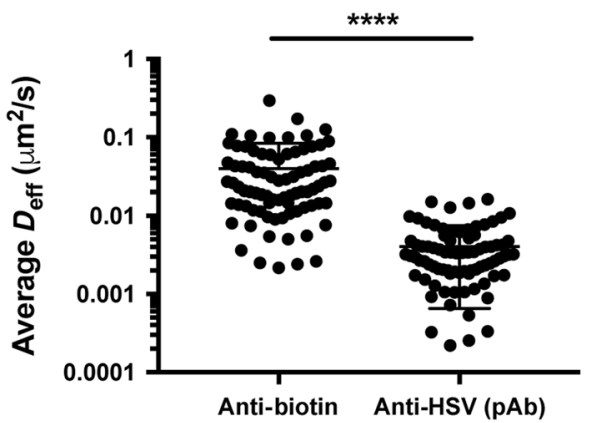

d

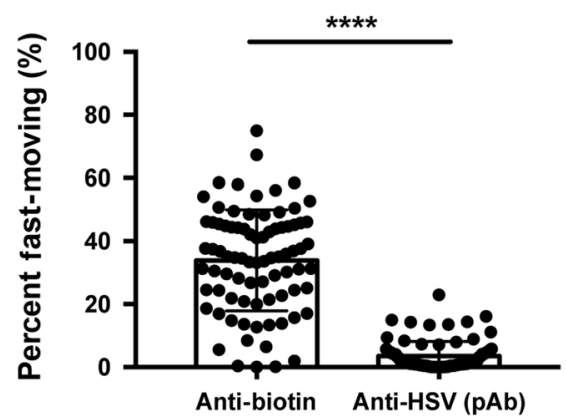

Fig. 1 HSV-1 mobility as a function of exogenously added polyclonal anti-HSV-1 and or non-specific anti-Biotin IgG control. a Sample trace of fast-moving HSV-1 subpopulations, with $D_{\text {eff }} \geq 0.347 \mu \mathrm{m}^{2} / \mathrm{s}$. b Sample trace of trapped virus in CVM. c Ensemble-averaged $\left\langle D_{\text {eff }}\right\rangle$ and d percent of fast-moving viruses in CVM treated with $5 \mu \mathrm{g} / \mathrm{mL}$ pAb. Paired two-tailed $t$-test $\left({ }^{*} p<0.05,{ }^{* *} p<0.01,{ }^{* * *} p<0.001,{ }^{* * * *} p<0.0001\right.$, $n=82$.

characteristics and mechanical (rheological) properties of mucus to factors such as menstrual cycle ${ }^{15-17}$, exogenous hormones ${ }^{17,18}$ and the vaginal microbiota. ${ }^{19,20}$ Indeed, the dense community of commensal microbiota that populate CVM not only varies considerably between women and within the same woman over time, but also directly impacts the biophysical properties of CVM and its diffusional barrier properties against viruses. ${ }^{21-23}$ For instance, $G$. vaginalis, the predominant microbe in bacterial vaginosis (BV), can secrete sialidase enzymes that cleave sialic acid sugars from mucins, leading to a marked loss in mucus viscoelasticity ${ }^{20,24,25}$ and is associated with an elevated risk for acquiring sexually transmitted infections (STIs). The innate diffusional barrier properties of CVM against HIV were previously shown to correlate to the vaginal microbiota. ${ }^{19,21,26}$

In order for this muco-trapping effector function to protection against vaginal infections, it is essential that this mechanism of vaginal immunity is conserved not only across the menstrual cycle, but also among women possessing different microbiota. ${ }^{6,27}$ To test the breadth and consistency of this trapping function, we collected fresh and undiluted CVM secretions from six women with different microbiota and contraceptive use across their menstrual cycle, and employed high-resolution multiple particle tracking to quantify the diffusion rates of hundreds of individual fluorescent HSV-1 virions in CVM treated with different antibodies (Abs). We found the barrier properties of CVM against HSV to be consistently reinforced by the presence of HSV-specific IgG in CVM.

\section{RESULTS}

Exogenous polyclonal and monoclonal antibodies against herpes simplex virus Type I (HSV-1) effectively trap HSV-1 in the CVM

In a previous study with seven CVM specimens from unique donors, we showed that exogenously added polyclonal antibodies (pAb) against HSV-1, purified from intravenous immunoglobulin, effectively trapped virions in $\mathrm{pH}$-neutralized mucus even at subneutralizing levels. In contrast, HSV readily penetrated the same CVM specimens in the absence of HSV-specific antibodies. ${ }^{14}$ We first sought to replicate our original finding that exogenously added anti-HSV-1 pAb would similarly trap HSV-1 in CVM. We mixed HSV-1 virions expressing a VP22-GFP (green fluorescent protein) tegument protein construct, packaged at high copy numbers while maintaining native viral envelope integrity $(\mathrm{d} \sim 180$ $\mathrm{nm}$ ) into undiluted, $\mathrm{pH}$-neutralized CVM treated with different $\mathrm{Ab}$, and performed multiple particle tracking to quantify the mobility of hundreds of individual viruses in each condition.

In 82 CVM specimens collected from six donors, the addition of anti-HSV-1 pAb to a final concentration of $5 \mu \mathrm{g} / \mathrm{mL}$ in CVM decreased the ensemble-averaged effective diffusivities $\left(\left\langle D_{\text {eff }}\right\rangle\right)$ for HSV-1 by tenfold, compared with anti-biotin IgG control, in good agreement with our previous observations (Fig. 1). ${ }^{14}$ There is substantial heterogeneity in the mobility of individual virions, particularly in native, untreated CVM; the same CVM specimen frequently contains both freely diffusing and immobilized virions. ${ }^{14,21,26}$ Virions that possess the greatest diffusivity (i.e., the most mobile fractions) by definition are more likely to diffuse across the mucus layer and infect the underlying epithelium before mucus is purged by natural clearance mechanisms. Thus, we sought to assess the effect of exogenous anti-HSV-1 pAb in limiting the fraction of HSV-1 that could most readily penetrate the CVM. We quantitatively defined the fast-moving subpopulation as virions that possess sufficient mobility to penetrate through a physiologically thick CVM layer $(50 \mu \mathrm{m})$ in one hour, which yielded a minimum $\left.D_{\text {eff }} \geq 0.347 \mu \mathrm{m}^{2} / \mathrm{s}\right){ }^{9}, 26,28$. The addition of anti-HSV-1 pAb reduced the fraction of fast-moving virions by $\sim$ tenfold, from $33.4 \% \pm 15.5 \%$ to $3.4 \pm 4.5 \%$. We evaluated the effectiveness of trapping at lower pAb concentrations in a subset of CVM specimens ( $n=26$, Fig. 2a, b). The average decreased $\sim$ threefold and $\sim$ fourfold in CVM containing 0.5 and $1 \mu \mathrm{g} / \mathrm{mL}$ anti-HSV-1 pAb compared to anti-biotin IgG control $\left(p<0.01^{* *}\right)$. This translated into a decrease in the fraction of "fast-moving" virions from $30.2 \pm 13.4 \%$ to $14.4 \pm 9.9 \%$ and 11.6 $\pm 11.6 \%$, respectively $\left(p<0.0001^{* * * *}\right)$.

Since monoclonal antibodies ( $\mathrm{mAb}$ ) are far easier and cheaper to manufacture and characterize than $\mathrm{pAb}$, we next investigated whether mAb against HSV-1 gD could immobilize the virions in CVM similarly to pAb. ${ }^{29}$ We produced two variants of the same anti-gD $\operatorname{lgG}_{1} \mathrm{mAb}$, one in mammalian cells (HSV8-293), and the other in tobacco plants i.e., Nicotiana benthamiana, engineered with human glycosylation pathways (HSV8-N). ${ }^{30}$ We were able to directly compare the trapping potency of all three $A b$ in the same CVM specimen in 70 of the 82 CVM specimens collected. In general, both mAb were slightly less effective at trapping HSV-1 than pAb, with $<D_{\text {eff }}>$ of $0.0057 \pm 0.005 \mu \mathrm{m}^{2} / \mathrm{s}, 0.0065 \pm 0.0065 \mu \mathrm{m}^{2} / \mathrm{s}$ and $0.004 \pm$ $0.003 \mu \mathrm{m}^{2} / \mathrm{s}$ in HSV8-293, HSV8-N, and pAb-treated CVM (final concentration $5 \mu \mathrm{g} / \mathrm{mL}$ ). The fast-moving populations were reduced from $32.3 \%$ in untreated CVM to $7.5 \pm 6.3 \%, 8.2 \pm 6.6 \%$ and $2.7 \pm$ 3.4\% for HSV8-293, HSV8-N, and pAb, respectively (Fig. 2c,d). These results underscore the potential of using $m A b$ to reinforce the diffusional barrier of CVM against specific pathogens.

Antibody-mediated trapping appears to be independent of menstrual cycle phase and consistent across the menstrual cycle Properties of cervical mucus such as mucus thickness and viscoelasticity vary substantially across the menstrual cycle in response to hormonal changes. ${ }^{18,27}$ Because CVM originates 
a

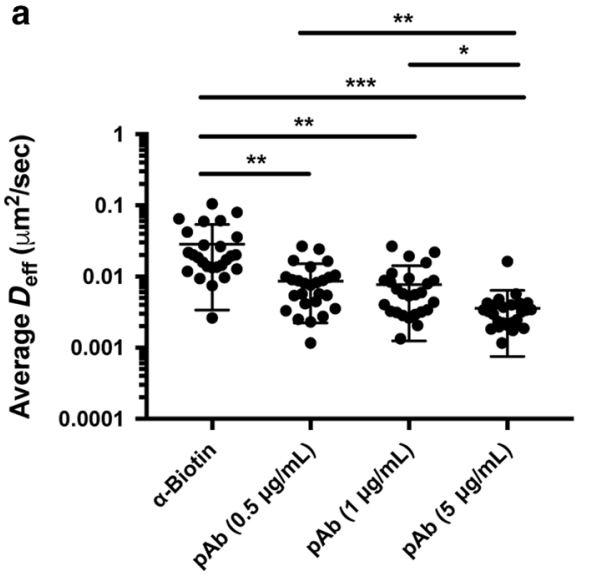

c

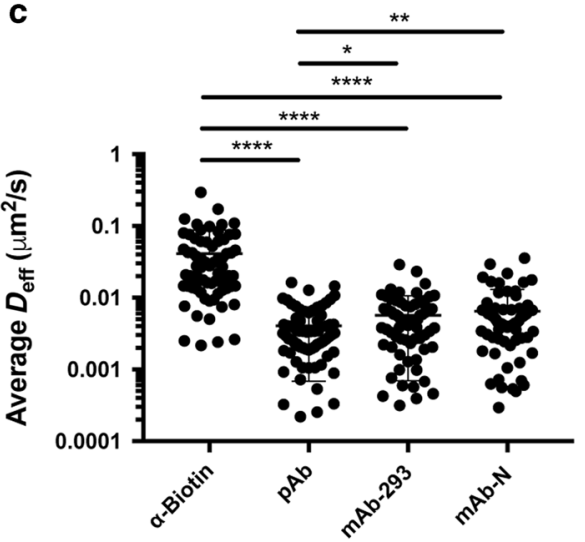

b

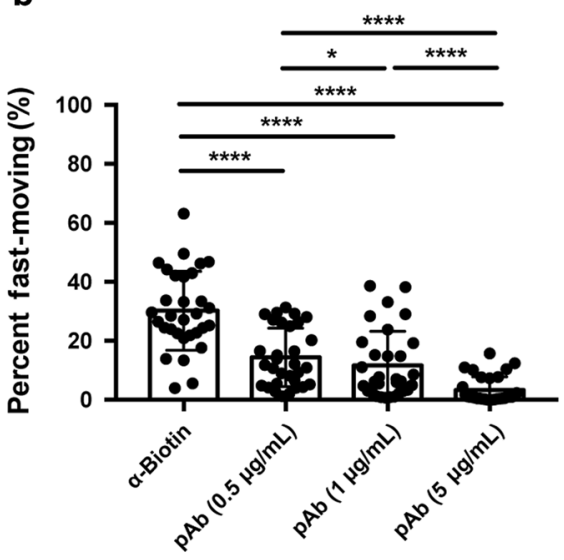

d

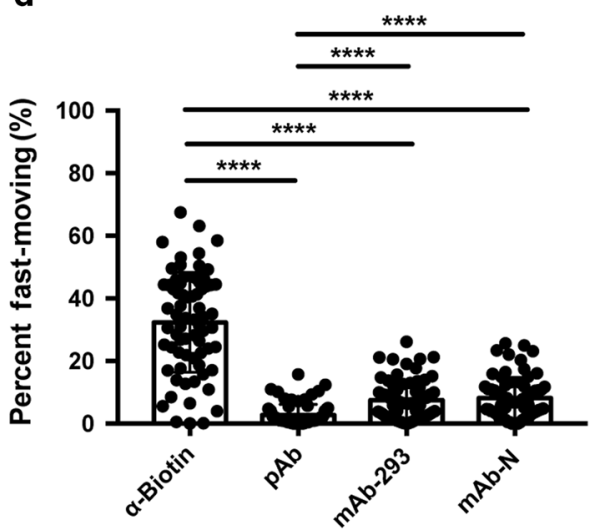

Fig. 2 HSV-1 mobility in CVM with exogenously added polyclonal anti-HSV-1 IgG (pAb) and monoclonal anti-HSV-1-gD. Comparison of IgG produced in Expi293 cells (mAb-293) and Nicotiana Benthamiana (mAb-N), and non-specific anti-Biotin IgG control. Polyclonal anti-HSV-1 was tested at a final concentration of 5,1 , or $0.5 \mu \mathrm{g} / \mathrm{mL}$. HSV8-293 and HSV8-N mAbs and non-specific anti-Biotin IgG control tested at $5 \mu \mathrm{g} / \mathrm{mL}$. a Ensemble-averaged $\left\langle D_{\text {eff }}\right\rangle$ and $\mathbf{b}$ percent of fast-moving virions as a function of increasing polyclonal anti-HSV-1 concentration. $\mathbf{c}$ Ensembleaveraged $\left\langle D_{\text {eff }}>\right.$ and $\mathbf{d}$ percent of fast-moving viruses in CVM treated with mAb-293 and mAb-N vs. pAb. All Ab concentrations are $5 \mu \mathrm{g} / \mathrm{mL}$. Repeated Measures One-Way ANOVA with multiple comparisons $\left({ }^{*} p<0.05,{ }^{* *} p<0.01,{ }^{* * *} p<0.001,{ }^{* * * *} p<0.0001\right)$. $N=28$ matched samples (A/B). $N=70$ matched samples, collected across six donors (C/D)

primarily from cervical mucus rather than from the secretion by vaginal epithelium, it is essential to investigate whether $\mathrm{pAb}$ and $m A b$ can effectively trap viruses in both luteal and follicular phase CVM. To examine this, we stratified the data from the $n=70$ specimens into either follicular or luteal phase, based on the day of the menstrual cycle when each sample was collected. We included CVM from women on hormonal birth control in this analysis, as many current hormonal contraceptives phase the level of hormone exposure throughout the treatment cycle, and a significant proportion of women still display ovarian activity and follicular development. Given it is more likely that women on hormonal contraceptive will engage in unprotected intercourse, it is critical for us to assess whether the effectiveness of IgGmediated trapping of viruses is conserved over time in women with hormonal contraceptives. ${ }^{31,32}$ Since none of the samples we collected displayed spinnbarkeit, the egg-white-like stretchy mucus that is the hallmark of ovulatory mucus, we excluded further stratifying the data into a third mid-cycle phase. While the potency of pAb was slightly greater relative to mAbs in the luteal phase $(n=38)$ compared to follicular phase $(n=32)$ samples, Abmediated trapping by mAbs was consistent across both phases, and there were no statistically significant differences in ensembleaveraged $\left\langle D_{\text {eff }}\right\rangle$ or percentage of fast-moving virions between the two mAb formats. Interestingly, the average $D_{\text {eff }}$ in the anti-Biotin IgG condition was approximately $50 \%$ lower in the luteal phase compared with follicular phase $\left(0.027 \pm 0.023 \mu \mathrm{m}^{2} / \mathrm{s}\right.$ vs. $0.057 \pm$
$0.06 \mu \mathrm{m}^{2} / \mathrm{s}$, ${ }^{* * *} p<0.0001$ ), possibly due to cervical mucus thickening in response to increased progesterone secretion (Fig. 3). ${ }^{16,18,19}$

We further investigated whether the trapping potency of pAb and $\mathrm{mAb}$ was consistent not only between two key phases of the menstrual cycle, but also across the 28-day cycle. We plotted the percentage of fast-moving virions and $\left\langle D_{\text {eff }}\right\rangle$ as a function of cycle day and tested for linearity using Pearson's correlation. Similar to above, there was a statistically significant trend in the anti-Biotin condition that suggests viruses were somewhat less mobile toward the end of the menstrual cycle than at the beginning $\left({ }^{* *} p<0.01\right)$. There was no statistically significant dependence of $\left\langle D_{\text {eff }}>\right.$ and fraction of fast-moving virions on cycle day for the various anti-HSV-1 Ab (Fig S1). Likewise, there were no statistically significant differences in the consistency of trapping potency between normally cycling donors and those taking birth control. Although the number of donors in our study was limited by our capacity to follow a larger group of women across several menstrual cycles, the results here are consistent with the notion that Ab-mediated trapping potency is likely independent of reproductive hormone levels in the female reproductive tract.

Antibody-mediated trapping potency of HSV-1 in CVM appears to be independent of vaginal community state type The vaginal microbiota composition is associated with susceptibility to a diverse array of sexually transmitted infections 
a

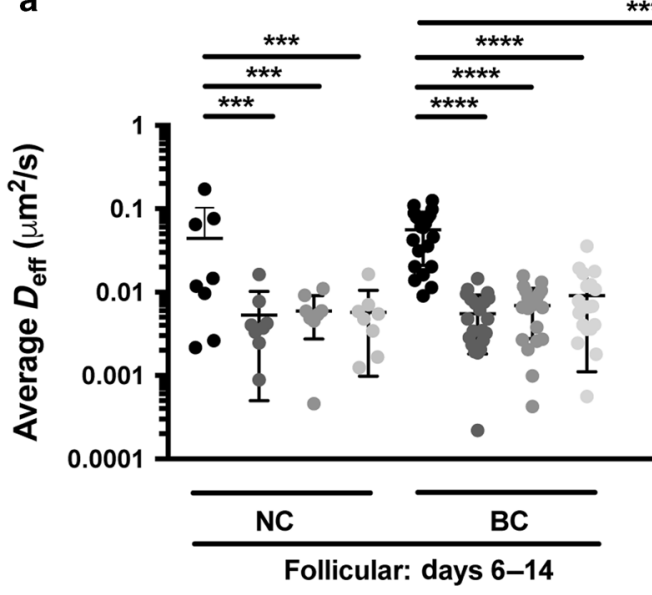

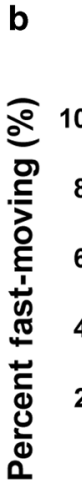
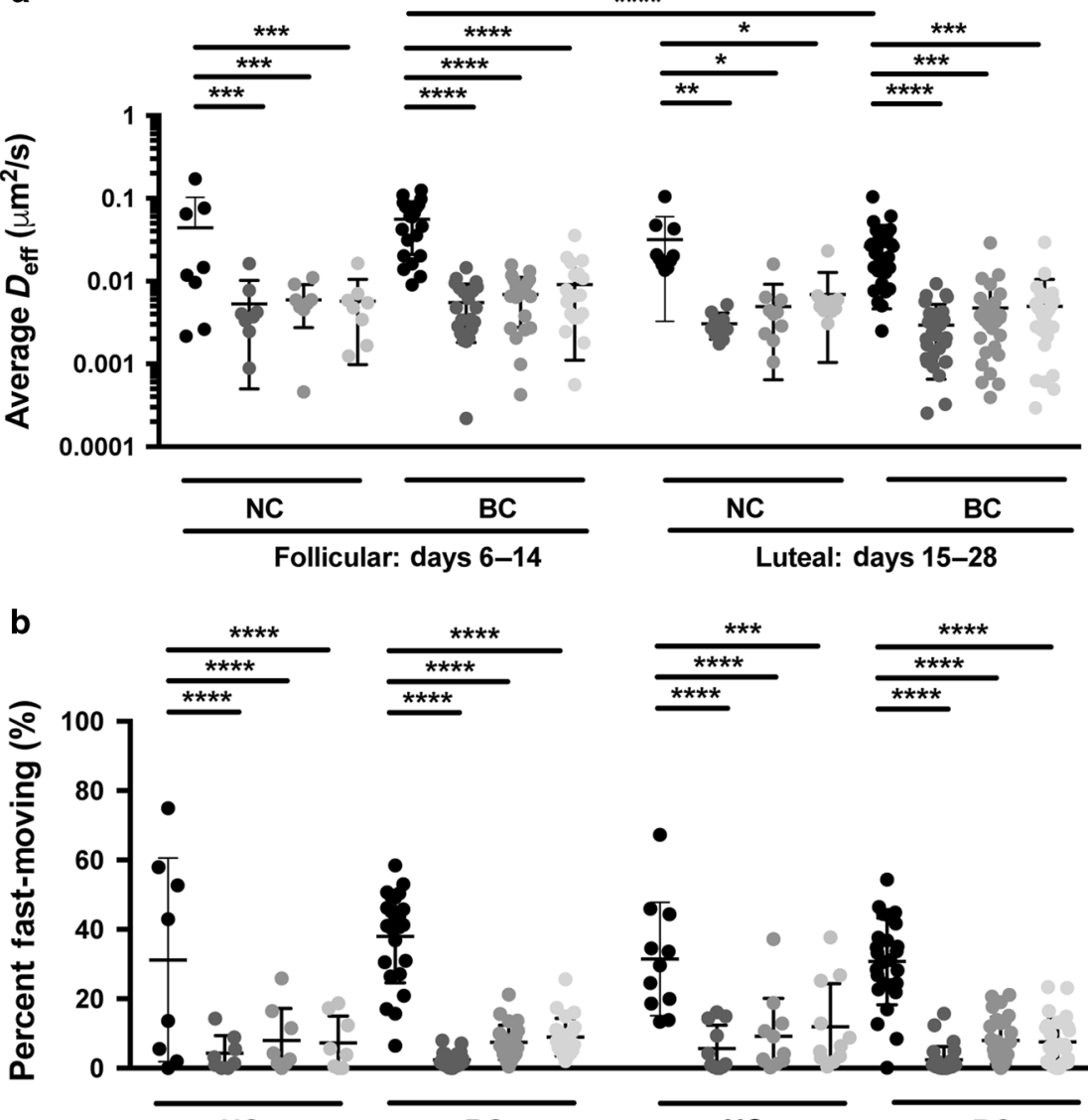

$* * * *$

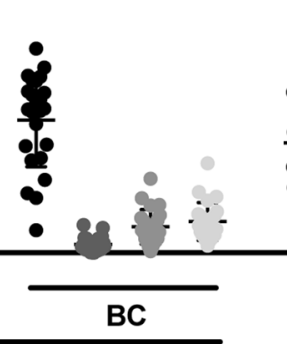

Follicular: days 6-14

- Anti-biotin - Anti-HSV (pAb)

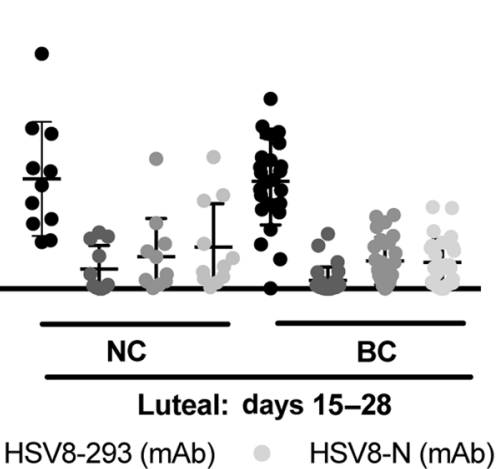

Fig. 3 Effect of menstrual cycle phase and birth control status on Ab-mediated trapping of HSV-1 in pH-neutralized CVM. Six donors were followed across a minimum of two menstrual cycles and reported the number of days post-menses; cycles were normalized to 28 days. CVM samples were grouped by the cycle phase and separated by birth control status. Samples obtained during the follicular phase were those collected 6-14 days post-menses, while those collected during the luteal phase were from 15-28 days post-menses. At least two samples were collected during the follicular phase and two during the luteal phase from each donor. All Abs were tested at $5 \mu \mathrm{g} / \mathrm{mL}$ and compared with anti-Biotin IgG in matched samples. a Average $\left\langle D_{\text {eff }}>\right.$ of virions in follicular versus luteal phase in NC versus BC donors. $\mathbf{b}$ Percentage of fastmoving virions (average $\left\langle D_{\text {eff }}>\geq 0.347 \mu \mathrm{m}^{2} / \mathrm{s}\right.$ ) by phase in follicular versus luteal phase in normally cycling (NC) versus donors on birth control (BC). Data was analyzed using Two-Way ANOVA with Sidak's multiple comparisons test. A $p \leq 0.05$ was considered statistically significant $\left({ }^{*} p<0.05,{ }^{* *} p<0.01,{ }^{* *} p<0.001,{ }^{* * *} p<0.0001\right) . N=32$ follicular phase samples, $N=38$ luteal phase samples

including herpes and HIV. ${ }^{25}$ Therefore, we were interested in how diverse vaginal microbiota may influence lgG-mediated trapping potency for monoclonal and polyclonal IgGs. We were able to characterize the microbial communities in 66 CVM specimens using $16 \mathrm{~s}$ rRNA gene sequencing and classified them into five community state types (CSTs). ${ }^{23}$ The majority of CVM specimens were classified as either CST I (L. crispatus-dominated, $n=18)$, CST III (L. iners-dominated, $n=22$ ), or CST IV (G. vaginalis-dominated, $n$ $=16)$, with a lower prevalence of CST II (L. gasseri-dominated, $n=$ 4) and CST V (L. jensenii-dominated, $n=6)$, in good agreement with previous findings (Fig S2, Table S1). ${ }^{23}$ Despite variations in the native CVM barrier to reduce virion mobility across CSTs, all Ab were able to effectively reduce the fraction of fast-moving virions across all CSTs (Fig S3). The addition of $5 \mu \mathrm{g} / \mathrm{mL}$ pAb to CVM reduced the fraction of fast-moving virions to $1.8 \% \pm 2.4 \%, 0.8 \% \pm$ $0.6 \%, 2.7 \% \pm 2.2 \%, 4.1 \% \pm 4.8 \%$, and $5.7 \% \pm 4.2 \%$ for CSTs I-V, respectively, compared to $28.4 \% \pm 11.5 \%, 20.0 \% \pm 19.8 \%, 37.3 \% \pm$ $17.1 \%, 38.5 \% \pm 14.9 \%$, and $31.5 \% \pm 17.3 \%$ for untreated CVM from CSTs I-V. Although slightly less potent than pAb, mAbs yielded similarly statistically significant decreases in both ensemble-averaged $\left\langle D_{\text {eff }}\right\rangle$ and fraction of fast-moving virions. There were no statistically significant differences in the trapping potency of either pAb or mAb between CSTs (Fig. 4). We further investigated the variations in IgG-mediated trapping of HSV within individual donors. Donors 21, 43, 57, and 58 had relatively consistent microbiota profiles across the time frame assessed, whereas donors 36 and 50 presented more dynamic and often changing microbiota (Fig. 5). Despite the differences in birth control status and fluctuations in microbiota, the various anti-HSV1 Abs consistently enabled effective trapping of HSV-1 in all six donors, and there were no statistically significant differences in the ability of Abs to slow virions between individuals (Fig. 5, Fig S4). Even in CVM with nearly $100 \%$ of the microbiota represented by $G$. vaginalis (donor F57), the fraction of fastmoving virions was substantially reduced from $38.1 \% \pm 9.1 \%$ in untreated control to $3.4 \% \pm 4.5 \%, 10.9 \% \pm 7.0 \%$, and $11.8 \% \pm 6.4 \%$ when treated with pAb, mAb-293, and mAb-N, respectively $(p<$ $\left.0.0001^{* * *}\right)$. This is comparable to $L$. crispatus (CST I)-dominant microbiota (F21), where the fraction of fast-moving virions was reduced from $28.8 \pm 15.1 \%$ to $2.6 \% \pm 2.8 \%$ by pAb, $5.4 \% \pm 4.0 \%$ by 
**

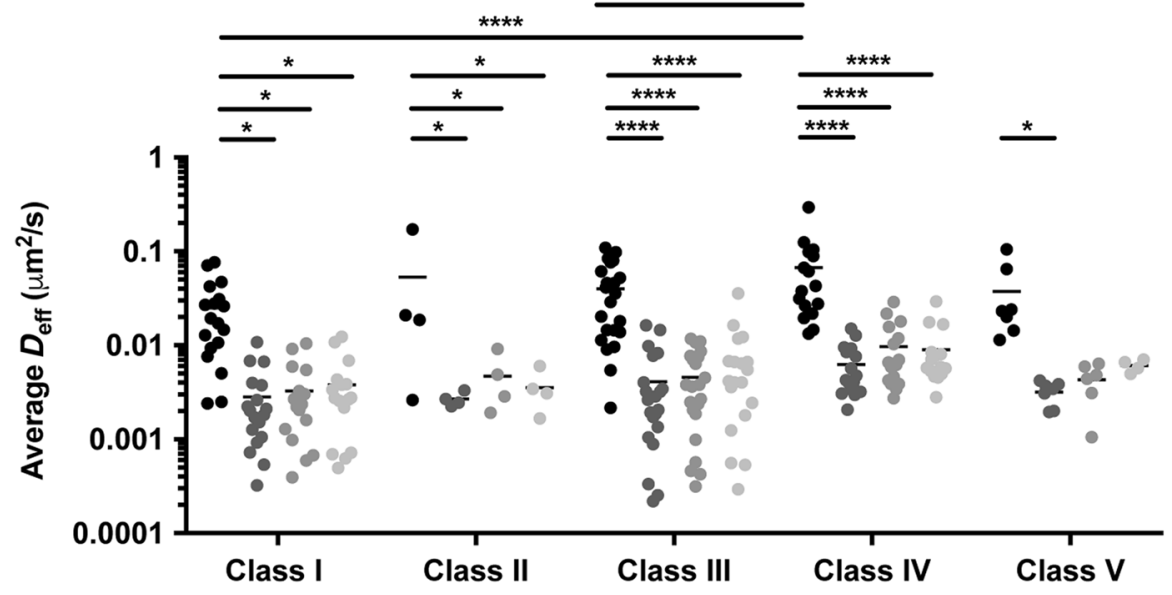

b

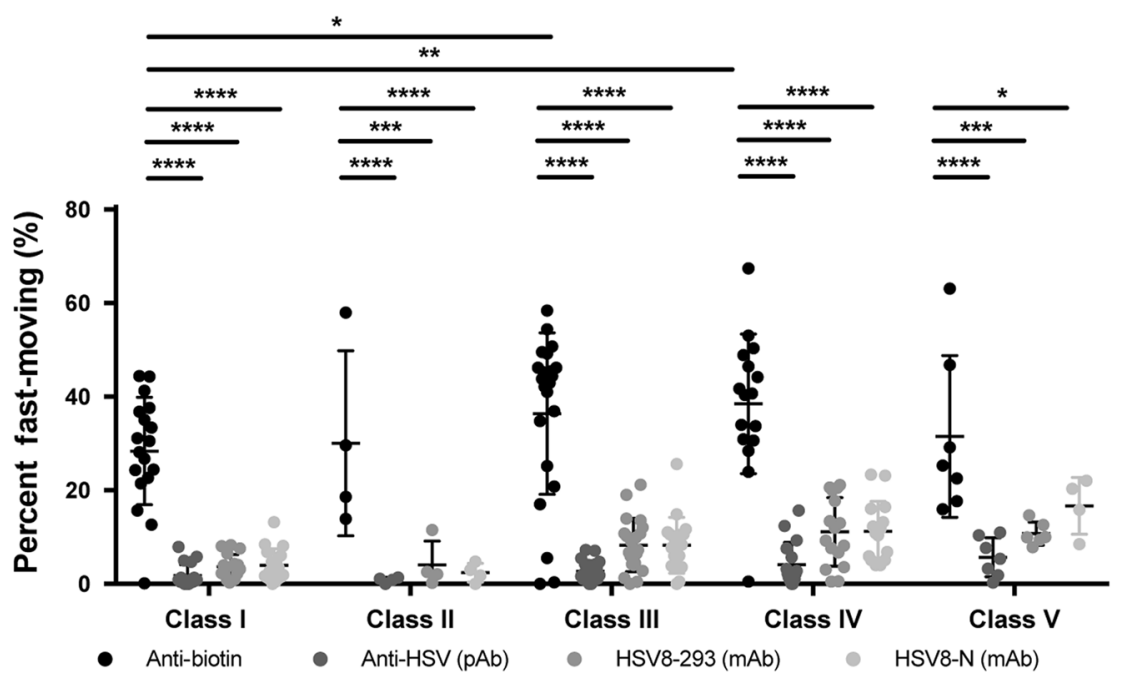

Fig. 4 Ab-mediated trapping of HSV-1 as a function of microbial Community State Type (CST). Samples were sequenced with $16 \mathrm{~s}$ rRNA whole-genome sequencing analysis and classified into CSTs depending on the predominant microbial species, according to the following groups: Class I (L.crispatus, $n=18)$, Class II (L.gasseri, $n=4)$, Class III (L.iners, $n=22)$, Class IV (G.vaginalis, $n=16)$, or Class $\vee(L . J e n s e n i i, ~ n=6){ }^{23}$ After classification, HSV-1 mobility was compared for each Ab condition. a Ensemble-averaged $\left\langle D_{\text {eff }}>\right.$ in CVM treated with different $A b$ and $\mathbf{b}$ percent fast-moving viruses $\left(D_{\text {eff }}\right) \geq 0.347 \mu \mathrm{m}^{2} / \mathrm{s}$. Two-Way ANOVA with multiple comparisons (Tukey's Multiple Comparison's Test) ( ${ }^{*} p<0.05$, $\left.{ }^{* *} p<0.01,{ }^{* * *} p<0.001,{ }^{* * * *} p<0.0001\right)$

HSV8-293, and $5.2 \% \pm 4.3 \%$ by HSV8-N $\left(p<0.0001^{* * * *}\right)$. There was no statistically significant difference in virion mobility in the presence of HSV-specific IgG between these CSTs (Fig S3).

\section{DISCUSSION}

STIs are pandemic worldwide; with the exception of HPV, there are no effective vaccines against virtually all common STIs including Herpes, HIV, Neisseria gonorrhoeae (GC), Chlamydia trachomatis, and syphilis. Given the longstanding challenge in eliciting durable immunity against a variety of STIs, some investigators are turning to passive immunization of the cervicovaginal environment-whereby protective $A b$ are delivered locally in the female reproductive tract-as a strategy to prevent STI transmission. $^{33,}{ }^{34}$ In addition to protection by neutralization ${ }^{35}$ and other classical effector functions, topically dosed $A b$ can facilitate protection by trapping virions in the mucus. For vaginal transmission, viruses must penetrate mucus secretions to reach target cells; indeed, many sexually transmitted viruses, including HIV, HSV, and HPV, can quickily diffuse and infect their target cells through CVM. $8,13,21,26$ Reinforcing the CVM barrier to trap pathogens in mucus thus represents an obvious strategy to prevent STIs. We found here that this unique muco-trapping $A b$ function is consistently observed both across the menstrual cycle, as well as in different women with varying microbiota composition. The consistency of this muco-trapping function further underscores its likely physiological importance and highlights passive immunization of the cervicovaginal environment as a promising strategy to protect against diverse STIs.

The potential muco-trapping effector function of IgG remains under-recognized, and rarely harnessed in the development of prophylaxis against STIs. Trapping in mucus can protect by directly limiting the flux of virions arriving at target cells in the epithelium, facilitating more complete inactivation by innate and adaptive immune mechanisms, and enabling more complete elimination by natural mucus clearance mechanisms. ${ }^{3}$ By preventing viruses from reaching target cells, Ab-mucin interactions may directly block infections at the portal of entry before initial infections even occur, as observed in our mouse Herpes transmission studies. ${ }^{14}$ This feature may be especially important against STIs that remain difficult to cure or eliminate once infection is established. ${ }^{7}$ Trapping pathogens in CVM is also likely complementary to current prophylactic approaches such as microbicides and PrEP, as combining different approaches may lead to synergistically 

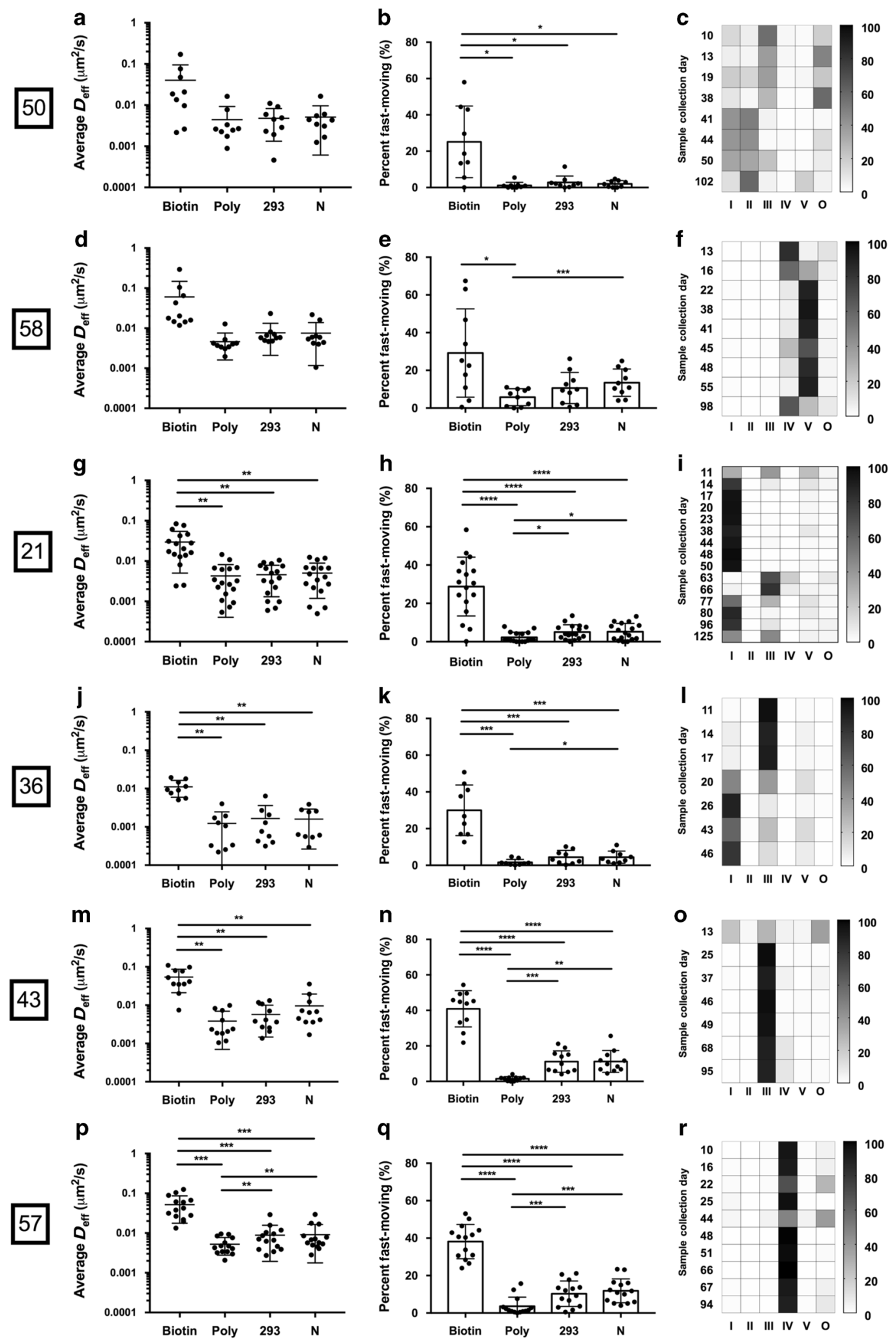

Fig. 5 Potency of Ab-mediated trapping in six different individuals across multiple menstrual cycles. Donors 50 and 58 were normally cycling a--f, while donors $21,36,43$, and 57 were on birth control g-r. Donor samples collected across multiple menstrual cycles were grouped to determine whether there was consistency in trapping potency within an individual, based on ensemble-averaged $\left\langle D_{\text {eff }}>(\mathbf{a}, \mathbf{d}, \mathbf{g}, \mathbf{j}, \mathbf{m}, \mathbf{p})\right.$ and percentage of fast-moving viruses $\mathbf{b}, \mathbf{e}, \mathbf{h}, \mathbf{k}, \mathbf{n}, \mathbf{q}$. Donor ID is presented on the left side of each set. Heat maps (C, f, i, I, $\mathbf{g}, \mathbf{r})$ show the proportion from CST I to CST V out of a total $100 \%$ of the sequenced microbiome, illustrating the inherent diversity of microbial populations both within an individual and across the menstrual cycle. Sample collection days are indicated on the left side of each heatmap. Microbes outside of CST I-V were classified as 'Other' $\mathbf{O}$. Heatmap scale ranges from 0 (white) to $100 \%$ (black). Data was analyzed using One-Way ANOVA with Tukey's Multiple Comparison's test $\left({ }^{*} p<0.05,{ }^{* *} p<0.01,{ }^{* * *} p<0.001,{ }^{* * * *} p<0.0001\right)$ 
enhanced protection. Finally, a large fraction of trapped virions will be rapidly eliminated via post-coital discharge, which usually occurs within minutes, suggesting that a comparable fraction of infectious virions will be cleared from the cervicovaginal environment if they are trapped in the discharge. Trapping virions in cervicovaginal mucus is likely to be especially important for preventing vaginal HIV, HSV, and HPV transmission, since the rate of transmission of these viruses is already quite low. ${ }^{3}$ The requirement for many intercourse events before a successful transmission event implies that few, if any, infectious virions actually reach target cells per intercourse. Therefore, reducing the fraction of virions that reach target cells will likely lead to a proportional reduction in transmission of infection.

The native barrier properties of CVM are directly influenced by the vaginal microbiota. For instance, we have shown that, unlike CVM from women with $L$. crispatus, CVM from women with $L$. iners and G. vaginalis-dominated microbiota, microbiota lacking a significant amount of Lactobacillus species, were unable to effectively immobilize HIV at native $\mathrm{pH}^{21}$ This in turn suggests that women with $L$. iners or $G$. vaginalis-dominant vaginal microbiota are potentially at greater risks of acquiring HIV, which has been confirmed by a number of epidemiological studies for a multitude of STIs. ${ }^{25,}{ }^{36}$ It is possible that the compromised barrier properties of CVM with dominant $L$. iners and $G$. vaginalis reflects a breakdown of mucins that could also compromise the ability for virus-bound Ab to crosslink with mucins. Indeed, G. vaginalis was thought to increase risks of STI transmission by overexpression of sialidases that can cleave terminal sialic acids and break down the mucin matrix of CVM, in addition to increasing the number of target cells in the epithelium due to its pro-inflammatory nature. ${ }^{16,}$ 20, 37, 38 Encouragingly, we found that both pAb and mAb were able to enhance the barrier properties of CVM against HSV across CVM from a diverse spectrum the vaginal microbiota, including $G$. vaginalis-dominated vaginal microbiota. These results suggest that harnessing $A b$-mucin interactions may help reduce STI transmission in women at the greatest risks of acquiring STI. ${ }^{24,} 35$

Cyclic fluctuations in estrogen and progesterone across the menstrual cycle are known to induce macroscopic changes in color, consistency, and viscosity of cervical mucus ${ }^{15,17,18}$; these changes are particularly important for enabling or preventing sperm ascension into the upper tract. Nevertheless, the potential variations of the barrier properties of CVM as a result of hormoneinduced fluctuations of CVM remains poorly understood, particularly for barrier properties at the length scale of viruses. Here, we found that independent of the phase of the menstrual cycle and use of hormonal contraceptive, HSV-binding Abs were able to specifically reinforce the barrier properties of CVM against HSV-1. Importantly, while genital herpes is conventionally associated with HSV-2, HSV-1 actually causes vaginal Herpes with comparable frequency, likely due in part to shifts in oral-genital sexual practices. ${ }^{39}$ In addition, the two HSV serotypes share significant sequence homology in their protein-coding regions $(83 \%)$, including neutralizing epitopes such as $\mathrm{gD}(85 \%$ sequence homology). ${ }^{39}$ Indeed, our monoclonal antibody binds both HSV1 and HSV-2, which is the reason why it is currently in clinical trials for vaginal Herpes prevention (NCT02579083). ${ }^{40}$ Further, while the number of donors in our study was limited, we speculate that the consistent manner with which we observed Ab-mediated reinforcement of the mucosal defense may be an evolutionary trait in humans: unlike most mammals, copulation frequently occurs not just during ovulation but across the entire menstrual cycle in humans, necessitating the need for protection against STIs beyond the mid-cycle.

For translational development, the batch-to-batch nature of pAb preparations is likely too expensive and too low throughput to address public health demands. ${ }^{33,41}$ Instead, passive immunization of the cervicovaginal environment will most likely rely on the scalable and increasingly economical processes of $\mathrm{mAb}$ production. Here, we found that the trapping potencies of $m A b$, regardless of whether they were produced in mammalian cells or glycan-modified Nicotiana benthamiana, were slightly reduced compared to pAb. This difference is likely attributed to a difference in the number of $A b$ bound to each virion. Compared to $\mathrm{mAb}$ that only binds to a specific epitope on the $\mathrm{gD}$ glycoprotein, pAb purified from whole HSV will include Ab that binds to all major glycoprotein epitopes on the virion surface, including $\mathrm{gB}, \mathrm{gC}, \mathrm{gG}$, and $\mathrm{gH}$, and thus achieve greater number of bound $\mathrm{Ab}$ than $\mathrm{mAb}$ at the same Ab concentrations. ${ }^{42}$ This suggests the slightly lower muco-trapping potency of mAb vs. pAb should be readily overcome by targeting epitopes that are present in high numbers on the pathogen surface or by simply including multiple different mAbs against the same pathogen. Indeed, this approach is increasingly utilized in mAbbased antivirals, such as ZMapp ${ }^{T M}$, a formulation of cocktail of three distinct $\mathrm{mAb}$ against Ebola. ${ }^{43}$ The use of multiple mAbs targeting different regions of the same epitope, or different epitopes on the same virus, would also limit escape mutants of the viruses. $^{29}$

A longstanding challenge that has limited applications of passive immunization is the large dose of Ab systemically necessary to facilitate protection at mucosal surfaces, which has translated into exceptionally high vaccination costs. ${ }^{44,} 45$ Nevertheless, a confluence of factors may enable cost-effective passive immunization of the vagina in the not-too-distant future. First, passive immunization has historically been attempted through systemically administered $\mathrm{Ab}$ rather than topical delivery. ${ }^{6}, 44$ Since the female reproductive tract is coated with far smaller volumes of CVM $(\sim 1-2 \mathrm{~mL})$ than the volume of blood in circulation $(\sim 5 \mathrm{~L})$, protective concentrations can thus be readily achieved with far lower amounts of Ab by directly delivering locally in the vagina, potentially resulting in cost savings. ${ }^{29,} 41,45$ Second, advances in drug delivery technologies have made it possible to achieve sustained release of $A b$ from vaginal rings. ${ }^{29}$ Third, the cost of $A b$ production has fallen greatly over the past two decades due to advances in bioprocessing technology, both in mammalian culture systems and the introduction of less common production systems, such as Nicotiana-produced mAb evaluated here. Large-scale mAb production costs were projected at $\sim \$ 100 / \mathrm{g}$ in 2010 , and will likely continue to fall in the years ahead. ${ }^{46,47}$ Since as little as tens of micrograms of mAb per day may be adequate to confer protection, it is theoretically possible to achieve passive immunization of the vagina and other mucosal surfaces that are costeffective, particularly for infections that currently lack effective prophylaxis. Lower costs would likely be achieved by further improvements in bioprocessing, as well as further optimization of Ab-mucin affinity. ${ }^{9}$

The number of specimens and donors we could include in this study is limited by the throughput of particle tracking experiments that allow us to capture and quantify the mobility of hundreds of individual virions in each specimen. Unlike most techniques in biology, there are no real-time, objective readouts in particle tracking experiments. Instead, particle tracking involves extensive, careful analysis to accurately extract quantitative motion from videos, due to the need to visually inspect and remove false positive and false negative traces. This is particularly true with videos capturing individual viruses in biological environments that inevitably suffer from low signal-to-noise contrasts. Despite these limitations, the consistencies of IgG-mediated trapping repeated observed in numerous distinct specimens from six women here, and in 11 subjects (each sampled once at a random time point) in our earlier study is consistent with the notion that lgGmucin crosslinking is a broadly conserved effector mechanism that is likely consistently observed in women both across the menstrual cycle, as well as in women with diverse vaginal microbiota. Our study here motivates further study with a much larger cohort. 


\section{METHODS AND MATERIALS}

CVM collection and characterization

CVM was collected and characterized as previously described. ${ }^{26}$ Detailed methods are provided in Supplementary Materials and methods.

\section{Ethics statement}

Written informed consent of participants was obtained after the nature and possible consequences of the study were explained. All studies were performed in accordance with a protocol approved by the Institutional Review Board of the University of North Carolina at Chapel Hill (IRB 10-1817).

\section{Culture and purification of fluorescent HSV-1}

The HSV-1 mutant $166 \mathrm{v}$ encoding a VP22-GFP tegument protein packaged into HSV-1 at high copy numbers was kindly provided by Richard Courtney and used in all microscopy and ELISA studies. The addition of GFP to the VP22 protein has no deleterious effects on viral replication, and the fluorescence of $166 \mathrm{v}$ was consistently more intense than that of HSV-1 mutants encoding other GFP fusion proteins. ${ }^{48}$ Virus was expanded by a multiplicity of infection (MOI) of three on confluent monolayers of HaCat cells (Addex Biotech, San Diego, CA) maintained in Dulbecco's modified Eagle's medium (Life Technologies, Grand Island, NY) supplemented with $5 \%$ fetal bovine serum, $1 \times$ L-glutamine, and $1 \times$ Penicillin/ Streptomycin. Culture medium was collected $16-18 \mathrm{~h}$ post infection and centrifuged at $1000 \times \mathrm{g}$ for $5 \mathrm{~min}$ to remove cell debris. The resulting supernatant was split into $30-\mathrm{mL}$ aliquots and precipitated overnight with a PEG/salt solution. Briefly, $10 \mathrm{~mL}$ of $1.55 \mathrm{M} \mathrm{NaCl}$ was added to $30 \mathrm{~mL}$ of crude virus supernatant, followed by $10 \mathrm{~mL}$ of $40 \%$ PEG 8000 (Sigma, St. Louis, MO). After overnight incubation at $4{ }^{\circ} \mathrm{C}$, the virus/PEG solution was centrifuged at $2555 \times g$ at $4{ }^{\circ} \mathrm{C}$ for $1 \mathrm{~h}$. The virus pellet was resuspended in $1 \times$ PBS (phosphate buffered saline) and centrifuged through a continuous $20-50 \%(\mathrm{w} / \mathrm{w})$ sucrose in PBS gradient for $1 \mathrm{~h}$ at $74,119 \times g$. The resulting virus band was further purified by diluting $1: 5$ in PBS, layered over $30 \%(w / w)$ sucrose in PBS, and centrifuging for $1.5 \mathrm{~h}$ at $83,472 \times g$ to pellet. Purified virus pellet was resuspended in PBS and stored as single-use aliquots at $80^{\circ} \mathrm{C}$.

Preparation and characterization of polyclonal anti-HSV-1 IgG Polyclonal anti-HSV-1 lgG was purified from intravenous immunoglobulin (IVlg, Privigen ${ }^{\circledR} ; \geq 98 \%$ lgG; CSL Behring, King of Prussia, PA) by affinity column purification. Detailed methods are provided in Supplementary Materials and Methods.

Production of monoclonal HSV8 in 293 cells and Nicotiana benthamiana

HSV8 are produced in Expi293F cells and Nicotiana benthamiana as previously described. ${ }^{30}$ Detailed methods are provided in the Supplementary Materials and Methods.

Multiple particle tracking of HSV-1 in CVM

To mimic neutralization of CVM by alkaline seminal fluid, we titrated CVM to $\mathrm{pH}$ 6.8-7.2 using small volumes (3\% v/v) of $3 \mathrm{~N}$ $\mathrm{NaOH}$ and confirmed the $\mathrm{pH}$ by using a $\mathrm{pH}$ microelectrode (Microelectrodes, Bedford, NH) calibrated with $\mathrm{pH} \mathrm{4,} \mathrm{7,} \mathrm{and} 10$ buffers. Samples were either treated or untreated by the addition of known amounts of purified anti-HSV-1 lgG or control (antiBiotin) IgG pre-mixed with fluorescent virions (approximately $10^{8}-10^{9}$ particles $/ \mathrm{mL}$ ) and incubated at $37^{\circ} \mathrm{C}$ for $15-30$ min prior to adding to $20 \mu \mathrm{L}$ CVM in a custom-made glass chamber. Once mixed, Abs, virus, and CVM were incubated at $37^{\circ} \mathrm{C}$ for and additional 15-30 min prior to microscopy. Control beads consisted of red or green fluorescent 200-nm carboxyl-modified PS particles (Molecular Probes, Eugene, OR), either uncoated (PS; mucoadhesive) or covalently conjugated with a low molecular weight
(2 kDa), amine-functionalized PEG (Rapp Polymere, Tuebingen, Germany) to produce coated particles (PS-PEG; muco-inert) as previously described. ${ }^{28}$ Translational motions of the particles were recorded using an electron multiplying charge-coupled-device (EMCCD) camera (Evolve 512; Photometrics, Tucson, AZ) mounted on an inverted epifluorescence microscope (AxioObserver D1; Zeiss, Thornwood, NY) equipped with an Alpha Plan-Apo 100/1.46 numerical-aperture (NA) objective, an environmental (temperature and CO2) control chamber, and light-emitting diode (LED) light source (Lumencor Light Engine DAPI/GFP/543/623/690). Videos $(512 \times 512$ pixels, 16 -bit image depth) were captured with MetaMorph imaging software (Molecular Devices, Sunnyvale, CA) at a temporal resolution of $66.7 \mathrm{~ms}$ and spatial resolution of $10 \mathrm{~nm}$ (nominal pixel resolution, $0.156 \mu \mathrm{m} /$ pixel) for $20 \mathrm{~s}$. The tracking resolution was determined by tracking the displacements of particles immobilized with a strong adhesive, following a previously described method. ${ }^{49}$ Particle trajectories (xy locations) were obtained using Video Spot Tracker (VST) developed by Computer-Integrated Systems for Microscopy and Manipulation (CISMM) at UNC-CH (http://cismm.cs.unc.edu/downloads). The threshold parameter in VST was set using the Rosin threshold algorithm, ${ }^{50}$ and tracking was monitored and corrected by human intervention. Image contrast was adjusted to improve particle visibility, but the same contrast level was applied throughout the entire video and did not bias toward any particle population. Subpixel tracking resolution was obtained by determining the precise location of the particle centroid by light intensityweighted averaging of the neighboring pixels. Trajectories were analyzed using "frame-by-frame" weighting" ${ }^{51}$ in which mean squared displacements (MSD) and $D_{\text {eff }}$ are first calculated for individual particle traces. Averages and distributions are then calculated at each frame based on only the particles present in that frame before averaging across all frames in the movie. This approach minimizes bias toward faster-moving particle subpopulations. Trajectories of at least 40 particles per frame on an average were analyzed for each experiment, which typically corresponds to greater than 100 individual particle traces throughout the video and is consistent with sampling in our previous work. ${ }^{14,}{ }^{21}$ Trapped particles were defined by a $D_{\text {eff }}$ value of $.0316 \mu \mathrm{m}^{2} / \mathrm{s}$ at a value of $0.2667 \mathrm{~s}$ (time scale corresponding to a minimum trajectory length of 5 frames); for particles $200 \mathrm{~nm}$ in diameter and larger, this cutoff translates to particles moving a distance much less than their diameter within $0.2667 \mathrm{~s}$.

\section{S rRNA sequencing and analysis}

DNA extractions for microbiota analysis were performed as previously described ${ }^{22,23}$ on aliquots of the same CVM samples used for microscopic studies and other characterizations described. The method of Fadrosh et al. ${ }^{52}$ was used to analyze the vaginal microbiota composition and structure, and relied on amplification and sequencing on an Illumina MiSeq instrument (300-bp paired-end reads) of the V3-to-V4 regions of the $16 \mathrm{~S}$ rRNA gene. Sequence analyses and taxonomic assignments were performed using a custom pipeline freely available on GitHub (https://github.com/cwzkevin/MiSeq16S). The resulting taxonomic assignments are shown in Table S1. To assess whether the vaginal microbiota affected the barrier properties and $A b$ mediated trapping in CVM, we grouped the samples into Community State Types (CSTs) I-V according to the most dominant bacterial species within a sample. Samples were assigned to CSTs according to those identified by Ravel et.al. ${ }^{23}$ : $L$. crispatus-dominated (CST I), L. gasseri dominated (CST II), $L$. iners-dominated (CST III), a diverse set of strict or facultative anaerobic bacteria such as G. vaginalis (CST IV) and L. jenseniidominated (CST V). A sample grouped into a particular CST contained an average of $\sim 80 \%$ of the class-defining species (Figure S2). However, microbial populations between the samples can be extremely diverse. In this sample set, percentage 
of dominant bacterial species used to classify CST ranged from 34.26 to $99.9 \%$ of the total bacteria.

Statistical analysis

Statistical comparisons were performed using ANOVA with multiple comparisons between matched samples (Tukey's multiple comparison's test), using GraphPad Prism version 7.0 (GraphPad Software, La Jolla, CA). Follicular and luteal phases were compared with two-way ANOVA. Correlation between antiHSV-1 lgG-mediated trapping and cycle day was measured using Pearson's correlation coefficient ( $r$ ). Differences were deemed significant at an alpha of 0.05 . Results are reported as mean \pm standard deviation.

\section{ACKNOWLEDGEMENTS}

This work was supported by the National Institutes of Health (http://www.nih.gov/) grants R21Al093242 (S.K.L.), U19AI096398 (S.K.L.), and U19AI084044 (J.R.), a Diversity Supplement 1F32AI102535 (K.L.N.), The David and Lucile Packard Foundation (https:// www.packard.org/) 2013-39274 (S.K.L.), the Eshelman Institute of Innovation (http:// unceii.org/, S.K.L.), and startup funds from the University of North Carolina Eshelman School of Pharmacy (https://pharmacy.unc.edu/; S.K.L). The funders had no role in study design, data collection and analysis, decision to publish, or manuscript preparation.

\section{AUTHOR CONTRIBUTIONS}

H.A.S., K.L.N., and S.K.L. conceptualized and designed the experiments; K.L.N recruited study participants; H.A.S., K.L.N., C.E.H., F.L., M.S.H., and S.K.L. performed experiments; H.A.S., A.S., and S.K.L. performed data analysis; M.H.L., K.J.W., and L.Z. provided reagents; H.A.S. and S.K.L. wrote the paper; H.A.S., K.J.W., L.Z., J.R., and S.K.L. edited the paper.

\section{ADDITIONAL INFORMATION}

The online version of this article (https://doi.org/10.1038/s41385-018-0054-z) contains supplementary material, which is available to authorized users.

Competing interests: Intellectual property associated with harnessing antibody-mucin interactions described in part in this publication was developed at the University of North Carolina-Chapel Hill (UNC-CH), and has been licensed to Mucommune, L.LC. S.K.L. is a founder of Mucommune and currently serves as its interim CEO, board of director, and in the scientific advisory board. S.K.L. owns company stock; S.K.L.'s relationship with Mucommune is subject to certain restrictions under University policy. The terms of this arrangement are being managed by UNC-CH in accordance with its conflict of interest policies.

Publisher's note: Springer Nature remains neutral with regard to jurisdictional claims in published maps and institutional affiliations.

\section{REFERENCES}

1. Gipson, I. Mucins of the human endocervix. Front. Biosci. 6, D1245-D1255 (2001).

2. Kieweg S. L. Gravity-induced coating flows of vaginal gel formulations: In vitro experimental analysis J. Pharmaceut. Sci. 93, 2941-2952 (2014).

3. Nguyen, P. V. Innate and adaptive immune responses in male and female reproductive tracts in homeostasis and following HIV infection. Cell Mol. Immunol. 11, 410-427 (2014).

4. Cone, R. A. Barrier properties of mucus. Adv. Drug Deliv. Rev. 61, 75-85 (2009).

5. Cole, A. Innate host defense of human vaginal and cervical mucosae. Curr. Top. Microbiol Immunol. 306, 199-230 (2006).

6. Naz, R. K. Female genital tract immunity: distinct immunological challenges for vaccine development. J. Reprod. Immunol. 93, 1-8 (2012).

7. Kumamoto, Y. \& Iwasaki, A. Unique features of antiviral immune system of the vaginal mucosa. Curr. Opin. Immunol. 24, 411-416 (2012).

8. Chen, A. et al. Transient antibody-mucin interactions produce a dynamic molecular shield against viral invasion. Biophys. J. 106, 2028-2036 (2014).

9. Wessler, T. et al. Using computational modeling to optimize the design of antibodies that trap viruses in mucus. ACS Infect. Dis. 2, 82-92 (2016).

10. Fahrbach, K. M., Malykhina, O., Stieh, D. J. \& Hope, T. J. Differential binding of $\lg G$ and $\lg A$ to mucus of the female reproductive tract. PLoS One 8, e76176 (2013).
11. Wang, Y. Y. et al. Diffusion of immunoglobulin $\mathrm{G}$ in shed vaginal epithelial cells and in cell-free regions of human cervicovaginal mucus. PLoS One 11, e0158338 (2016).

12. Saltzman, W. M., Radomsky, M. L., Whaley, K. J. \& Cone, R. A. Antibody diffusion in human cervical mucus. Biophys. J. 66(2 Pt 1), 508-515 (1994).

13. Olmsted, S. S. et al. Diffusion of macromolecules and virus-like particles in human cervical mucus. Biophys. J. 81, 1930-1937 (2001).

14. Wang, Y. Y. et al. IgG in cervicovaginal mucus traps HSV and prevents vaginal herpes infections. Mucosal Immunol. 7, 1036-1044 (2014).

15. Sharif K. The Structure, Chemistry and Physics of Human Cervical Mucus, Vol. 2nd edn. (Blackwell Publishing Ltd, Oxford, 2006).

16. Moncla, B. J., Chappell, C. A., Debo, B. M. \& Meyn, L. A. The Effects of Hormones and Vaginal Microflora on the Glycome of the Female Genital Tract: CervicalVaginal Fluid. PLoS One 11, e0158687 (2016).

17. Chappell, C. A. et al. The effects of reproductive hormones on the physical properties of cervicovaginal fluid. Am. J. Obstet. Gynecol. 211, 226 e221-226 e227 (2014).

18. Aksoy, M., Guven, S., Tosun, I., Aydin, F. \& Kart, C. The effect of ethinyl estradiol and drospirenone-containing oral contraceptives upon mucoprotein content of cervical mucus. Eur. J. Obstet. Gynecol. Reprod. Biol. 164, 40-43 (2012).

19. Shukair, S. A. et al. Human cervicovaginal mucus contains an activity that hinders HIV-1 movement. Mucosal Immunol. 6, 427-434 (2013).

20. Moncla, B. J. et al. Impact of bacterial vaginosis, as assessed by nugent criteria and hormonal status on glycosidases and lectin binding in cervicovaginal lavage samples. PLoS One 10, e0127091 (2015).

21. Nunn, K. L. et al. Enhanced Trapping of HIV-1 by Human Cervicovaginal Mucus Is Associated with Lactobacillus crispatus-Dominant Microbiota. MBio. 6, e01084-01015 (2015).

22. Gajer, P. et al. Temporal dynamics of the human vaginal microbiota. Sci. Transl. Med. 4, 132ra152 (2012).

23. Ravel, J. et al. Vaginal microbiome of reproductive-age women. Proc. Natl Acad. Sci. USA 108(Suppl 1), 4680-4687 (2011).

24. Ma, B., Forney, L. J. \& Ravel, J. Vaginal microbiome: rethinking health and disease Annu. Rev. Microbiol. 66, 371-389 (2012).

25. Borgdorff, $\mathrm{H}$. et al. Cervicovaginal microbiome dysbiosis is associated with proteome changes related to alterations of the cervicovaginal mucosal barrier. Mucosal Immunol. 9, 621-633 (2016).

26. Lai, S. K. et al. Human Immunodeficiency Virus Type 1 Is Trapped by Acidic but Not by Neutralized Human Cervicovaginal Mucus. J. Virol. 83, 11196-11200 (2009).

27. Wira, C. R., Fahey, J. V., Rodriguez-Garcia, M., Shen, Z. \& Patel, M. V. Regulation of mucosal immunity in the female reproductive tract: the role of sex hormones in immune protection against sexually transmitted pathogens. Am. J. Reprod. Immunol. 72, 236-258 (2014).

28. Lai, S. K., Wang, Y. Y., Hida, K., Cone, R. \& Hanes, J. Nanoparticles reveal that human cervicovaginal mucus is riddled with pores larger than viruses. Proc. Natl Acad. Sci. USA 107, 598-603 (2010).

29. Whaley, K. J. \& Zeitlin, L. Antibody-based concepts for multipurpose prevention technologies. Antivir. Res. 100(Suppl), S48-S53 (2013).

30. Zeitlin, L. et al. Enhanced potency of a fucose-free monoclonal antibody being developed as an Ebola virus immunoprotectant. Proc. Natl Acad. Sci. USA 108, 20690-20694 (2011).

31. Klipping, C., Duijkers, I., Trummer, D. \& Marr, J. Suppression of ovarian activity with a drospirenone-containing oral contraceptive in a $24 / 4$ regimen. Contraception 78, 16-25 (2008).

32. Han, L., Taub, R. \& Jensen, J. T. Cervical mucus and contraception: what we know and what we don't. Contraception 96, 310-321 (2017).

33. Clementi, N. et al. Role and potential therapeutic use of antibodies against herpetic infections. Clin. Microbiol. Infect. 23, 381-386 (2017).

34. Yamamoto, H. \& Matano, T. Patterns of HIV/SIV Prevention and Control by Passive Antibody Immunization. Front Microbiol. 7, 1739 (2016).

35. Whaley, K. J. \& Mayer, K. H. Strategies for preventing mucosal cell-associated HIV transmission. J. Infect. Dis. 210(Suppl 3), S674-S680 (2014).

36. Gosmann, C. et al. Lactobacillus-deficient cervicovaginal bacterial communities are associated with increased HIV acquisition in young south African women. Immunity 46, 29-37 (2017).

37. Wiggins, R. H. S., Soothill, P. W., Millar, M. R. \& Corfield, A. P. Mucinases and sialidases: their role in the pathogenesis of sexually transmitted infections in the female genital tract. Sex. Transm. Inf. 77, 402-408 (2001).

38. Bertran, T. et al. Slight pro-inflammatory immunomodulation properties of dendritic cells by Gardnerella vaginalis: the "Invisible Man" of bacterial vaginosis? J. Immunol. Res. 2016, 9747480 (2016).

39. LeGoff, J., Pere, H. \& Belec, L. Diagnosis of genital herpes simplex virus infection in the clinical laboratory. Virol. J. 11, 83 (2014).

40. Zeitlin, L. et al. Topically applied human recombinant monoclonal $\lg G 1$ antibody and its $\mathrm{Fab}$ and $\mathrm{F}\left(\mathrm{ab} \mathrm{b}^{\prime}\right) 2$ fragments protect mice from vaginal transmission of HSV2. Virology 225, 213-215 (1996). 
1486

41. Fernandez-Romero, J. A. et al. Multipurpose prevention technologies: the future of HIV and STI protection. Trends Microbiol. 23, 429-436 (2015).

42. Uyangaa, E., Patil, A. M. \& Eo, S. K. Prophylactic and therapeutic modulation of innate and adaptive immunity against mucosal infection of herpes simplex virus. Immune Netw. 14, 187-200 (2014).

43. Qiu, X. et al. Reversion of advanced Ebola virus disease in nonhuman primates with ZMapp. Nature 514, 47-53 (2014).

44. Roth, K., Ferreira, V. H. \& Kaushic, C. HSV-2 vaccine: current state and insights into development of a vaccine that targets genital mucosal protection. Microb. Pathog. 58, 45-54 (2013).

45. Escolano, A., Dosenovic, P. \& Nussenzweig, M. C. Progress toward active or passive HIV-1 vaccination. J. Exp. Med. 214, 3-16 (2017).

46. Kelley, B. Industrialization of mAb production technology. mAbs. 1, 443-452 (2009).
47. Liu, H. F., Ma, J., Winter, C. \& Bayer, R. Recovery and purification process development for monoclonal antibody production. mAbs. 2, 480-499 (2014).

48. O'Hare, G. E. P. Live-cell analysis of a green fluorescent protein-tagged herpes simplex virus infection. J. Virol. 73, 4110-4119 (1999).

49. Apgar, J. et al. Multiple-particle tracking measurements of heterogeneities in solutions of actin filaments and actin bundles. Biophys. J. 79, 1095-1106 (2000).

50. Lai, Y. K. \& Rosin, P. L. Efficient circular thresholding. IEEE Trans. Image Process 23, 992-1001 (2014).

51. Wang, Y. Y., Nunn, K. L., Harit, D., McKinley, S. A. \& Lai, S. K. Minimizing biases associated with tracking analysis of submicron particles in heterogeneous biological fluids. J. Control Release. 220(Pt A), 37-43 (2015).

52. Fadrosh DW M. B., et al. An improved dual-indexing approach for multiplexed 16S rRNA gene sequencing on the Illumina MiSeq platform. Microbiome. 2, 6 (2014). 